\title{
First report on Meloidogyne chitwoodi hatching inhibition activity of essential oils and essential oils fractions
}

Jorge Miguel Silva Faria ${ }^{1}$ - Inês Sena ${ }^{1}$ - Bruno Ribeiro ${ }^{2}$ - Ana Margarida Rodrigues ${ }^{1}$. Carla Maria Nobre Maleita ${ }^{3}$. Isabel Abrantes ${ }^{4}$. Richard Bennett ${ }^{5}$.

Manuel Mota $^{2}$ - Ana Cristina da Silva Figueiredo ${ }^{1}$

Received: 23 September 2014/Revised: 16 March 2015/Accepted: 31 March 2015 Springer-Verlag Berlin Heidelberg 2015

Abstract The Columbia root-knot nematode (CRKN), Meloidogyne chitwoodi, is an EPPO A2 type quarantine pest since 1998. This nematode causes severe damage in economically important crops such as potato and tomato, making agricultural products unacceptable for the fresh market and food processing. Commonly used nematicidal synthetic chemicals are often environmentally unsafe. Essential oils (EOs) may constitute safer alternatives against RKN. EOs, isolated from 56 plant samples, were tested against CRKN hatching, in direct contact bioassays Some of the most successful EOs were fractionated and the hydrocarbon molecules (HM) and oxygen-containing

Electronic supplementary material The online version of thi article (doi:10.1007/s10340-015-0664-0) contains supplementary material, which is available to authorized users.

Communicated by M.B. Isman

A Ana Cristina da Silva Figueiredo acsf@fc.ul.pt molecules (OCM) fractions tested separately. 24 EOs displayed very strong hatching inhibitions $(\geq 90 \%)$ at $2 \mu \mathrm{L} \mathrm{mL}{ }^{-1}$ and were further tested at lower concentrations. Dysphania ambrosioides, Filipendula ulmaria, Ruta graveolens, Satureja montana and Thymbra capitata EOs revealed the lowest $\mathrm{EC}_{50}$ values $\left(<0.15 \mu \mathrm{L} \mathrm{mL}^{-1}\right)$. The main compounds of these EOs, namely 2-undecanone, ascaridol, carvacrol, isoascaridol, methyl salicylate, p-cymene and/or $\gamma$-terpinene, were putatively considered responsible for $\mathrm{CRKN}$ hatching inhibition. S. montana and T. capitata OCM fractions showed hatching inhibitions higher than $\mathrm{HM}$ fractions. The comparison of $\mathrm{EO}$ and corresponding fractions $\mathrm{EC}_{50}$ values suggests interactions between OCM and HM fractions against CRKN hatching. These species EOs showed to be potential environmentally friendly CRKN hatching inhibitors; nonetheless, bioactivity should be considered globally, since its HM and OCM fractions may contribute, diversely, to the full antihatching activity.

Keywords Columbia root-knot nematode - Dysphania ambrosioides - Filipendula ulmaria - Ruta graveolens. Satureja montana . Thymbra capitata 